\title{
Tracer particles sense local stresses in an evolving multicellular spheroid without affecting the anomalous dynamics of the cancer cells
}

\author{
Himadri S. Samanta ${ }^{\oplus},{ }^{1}$ Sumit Sinha $^{\oplus}{ }^{2}$ and D. Thirumalai ${ }^{1, *}$ \\ ${ }^{1}$ Department of Chemistry, University of Texas at Austin, TX 78712 \\ ${ }^{2}$ Department of Physics, University of Texas at Austin, TX 78712
}

(Dated: February 15, 2022)

\begin{abstract}
Measurements of local stresses on the cancer cells (CCs), inferred by embedding inert compressible tracer particles (TPs) in a growing multicellular spheroid (MCS), show that pressure decreases monotonically as the distance from the core of the MCS increases. How faithfully do the TPs report the local stresses in the CCs is an important question because pressure buildup in the MCS is dynamically generated due to CC division, which implies that the CC dynamics should be minimally altered by the TPs. Here using theory and simulations, we show that although the TP dynamics is unusual, exhibiting sub-diffusive behavior on times less than the CC division times and hyperdiffusive dynamics on in the long-time limit, they do not affect the long-time CC dynamics or the local CC stress distributions. The CC pressure profile within the MCS, which decays from a high value at the core to the periphery, is almost identical with and without the TPs. That the TPs have insignificant effect on the local stresses in the MCS implies that they are reliable reporters of the CC microenvironment.

PACS numbers:
\end{abstract}

The interplay between short-range forces and non-equilibrium processes arising from cell division and apoptosis gives rise to unexpected dynamics in the collective migration of cancer cells [1-9]. An example is the dynamics of cancer cells (CCs) in a growing multicellular spheroid (MCS), which is relevant in cancer metastasis $[7,8]$. Imaging experiments show that collective migration of a group of cells that maintain contact for a long period of time exhibits far from equilibrium characteristics [8,10-13]. The experimental studies have served as an impetus to develop simulations and theoretical models [14-19], which have given insights into the dynamics of collective motion in the MCS. Dynamics in a growing MCS is reminiscent of the influence of active forces in abiotic systems [20-22]. In a growing MCS, the analogue of active forces are self-generated [23], arising from biological events characterized by cell growth, division and apoptosis.

Although it has long been recognized that the stresses in the tumor interior are enhanced[24-26], which explained the reduction in proliferation, it is only recently that direct non-uniform pressure variations in MCS has been measured. Following the early pioneering experiments [24-26], several experimental studies that probe the local stresses or pressure on the CCs [27-32] have provided insights into the mechanism by which the CCs invade the extracellular matrix. The stresses within MCSs were measured [32] by embedding micron-sized inert deformable polyacrylamide beads, referred to as tracer particles (TPs). The reduction in the volume of the TPs in the presence of the colon carcinoma cancer cells, was used to estimate the strain on the TPs. From the calibrated stress-strain plot for the TPs, measured in the absence of the CCs, they obtained the stress value at the location of the TPs. By assuming that the stresses associated with the fluorescently labeled TPs faithfully report the pressure of the CCs, it was argued that the pressure propagates non-uniformly across the tumor. It was concluded that the TPs could be used as local stress probes or sensors.

The experiments raise an important question: What should be the characteristics of the TPs for them to be faithful sensors of stresses in an evolving MCS? This question emerges naturally from the experimental results [32, 33]. We believe that the following criteria must be satisfied : (a) Because in a growing MCS, pressure is dynamically generated predominantly by cell division, the TPs should have negligible effect on the CC dynamics. (b) The radial pressure profile in the MCS with and without the TPs must be similar. (c) Finally, the TPs should have negligible effect on the distribution of pressure on the cells across the MCS. In essence, the TPs should "observe" but not "disturb" the CC microenvironment.

In order to assess the influence of TPs on the fate of cancer cells, we used theory and simulations by embedding the TPs in an evolving MCS. We first show that the CCs, whose dynamics is sub-diffusive for $t \lesssim \tau$, exhibits super-diffusive behavior at long times with the MSD, $\Delta_{C C}(t) \sim t^{\alpha_{C C}}$. Surprisingly, $\alpha_{C C}$ does not change appreciably from the value in the absence of the TPs. Thus, criterion (a) described above is satisfied. We note parenthetically

\footnotetext{
*Electronic address: dave.thirumalai@gmail.com

$[\oplus]$ : Equal Contribution
} 

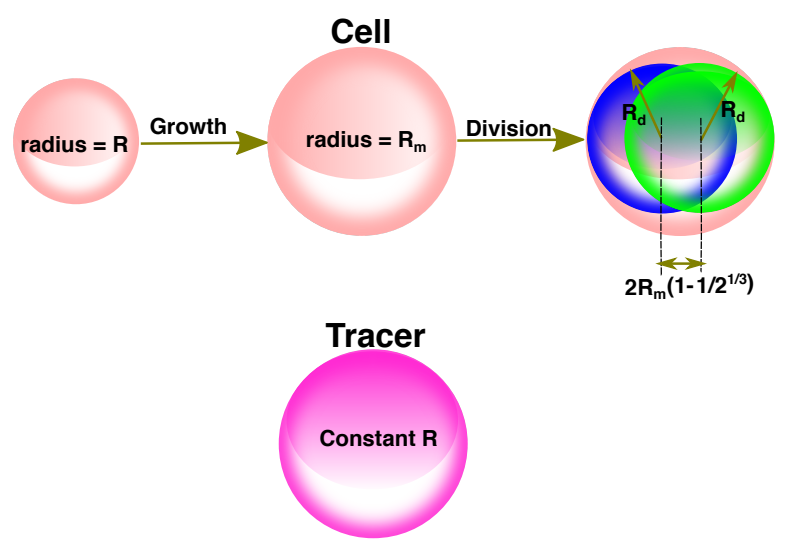

Figure 1: Difference between the CCs (pink sphere) and the TPs (magenta sphere). Cell division creates two daughter CCs (blue and green sphere), each with $R_{d}=\frac{R_{m}}{2^{1 / 3}}$. Their relative position is displaced by $2 R_{m}\left(1-\frac{1}{2^{1 / 3}}\right)$ with the orientation being random with respect to the sphere center.

that the motility of the TPs is unusual. On short times $\left(t \lesssim \tau\right.$, the cell division time) $\Delta_{T P} \sim t^{\beta_{T P}}$ with $\beta_{T P}<1$. In the long-time limit, $(t>\tau)$, the TPs undergo hyper-diffusive motion, $\Delta_{T P}(t) \sim t^{\alpha_{T P}}$ with $\alpha_{T P}>2$. We find that the pressure profiles vary radially across the MCS. The pressure is larger near the core and decreases as the boundary of the tumor is approached. Most importantly, the radial pressure profiles of the CCs with and without the TPs are virtually identical. We summarize that criteria (b) and (c) are satisfied, which shows that deformable gel-like TPs may be used as sensors of local stresses in the tumor.

\section{Results:}

The stresses in an evolving tumor are generated by dividing cancer cells. The stresses, created by active forces generated CC division, do not relax fast rapidly, especially when the tumor acquires has core structure. Because the CCs generate active forces through stochastic cell division, we first begin by describing the TPs and CCs, and how they affect each other.

Theoretical Considerations: We consider the dynamics of deformable inert TPs in a growing MCS in a dissipative environment by neglecting inertial effects. In contrast to the TPs, the CCs grow and divide at a given rate, and also undergo apoptosis (see Figure (1)). The CCs and TPs experience systematic short-ranged attractive and repulsive forces arising from the other CCs and TPs [14]. To model a growing MCS, we modify the density equation for the CCs phenomenologically by adding a non-linear source term, $\propto \phi\left(\phi_{0}-\phi\right)$, accounting for cell birth and apoptosis, and a nonequilibrium noise term that breaks the $\mathrm{CC}$ number conservation [34]. The noise, $f_{\phi}$, satisfies $<f_{\phi}(\mathbf{r}, t) f_{\phi}\left(\mathbf{r}^{\prime}, t^{\prime}\right)>=$ $\delta\left(\mathbf{r}-\mathbf{r}^{\prime}\right) \delta\left(t-t^{\prime}\right)$. The source term, $\propto \phi\left(\phi_{0}-\phi\right)$, represents the birth and apoptosis, with $\phi_{0}=\frac{2 k_{b}}{k_{a}}[34,35]$. The coefficient, $\sqrt{k_{b} \phi+\frac{k_{a}}{2} \phi^{2}}$ of $f_{\phi}$, is the noise strength, corresponding to number fluctuations.

In order to obtain analytic insights, we first derive suitable equations that describe the dynamics of the TPs and CCs. We introduce the density fields $\phi(\mathbf{r}, t)=\sum_{i} \delta\left[\mathbf{r}-\mathbf{r}_{i}(t)\right]$ for the CCs, and $\psi(\mathbf{r}, t)=\sum_{i} \delta\left[\mathbf{r}-\mathbf{r}_{i}(t)\right]$ for the TPs. A formally exact Langevin equation for $\phi(\mathbf{r}, t)$ and $\psi(\mathbf{r}, t)$ may be derived using the Dean's method [36] that accounts for diffusion and non-linear interactions. The equations for $\phi(\mathbf{r}, t)$ and $\psi(\mathbf{r}, t)$ are,

$$
\begin{aligned}
& \frac{\partial \psi(\mathbf{r}, t)}{\partial t}=D_{\psi} \nabla^{2} \psi(\mathbf{r}, t)+\nabla \cdot(\psi(\mathbf{r}, t) \mathbf{J})+\tilde{\eta}_{\psi}, \\
& \frac{\partial \phi(\mathbf{r}, t)}{\partial t}=D_{\phi} \nabla^{2} \phi(\mathbf{r}, t)+\nabla \cdot(\phi(\mathbf{r}, t) \mathbf{J})+\frac{k_{a}}{2} \phi\left(\frac{2 k_{b}}{k_{a}}-\phi\right)+\sqrt{k_{b} \phi+\frac{k_{a}}{2} \phi^{2}} f_{\phi}+\tilde{\eta}_{\phi}
\end{aligned}
$$

where $\mathbf{J}=\int_{\mathbf{r}^{\prime}}\left[\psi\left(\mathbf{r}^{\prime}, t\right)+\phi\left(\mathbf{r}^{\prime}, t\right)\right] \nabla U\left(\mathbf{r}-\mathbf{r}^{\prime}\right), \tilde{\eta}_{\psi}(\mathbf{r}, t)=\nabla \cdot\left(\eta_{\psi}(\mathbf{r}, t) \psi^{1 / 2}(\mathbf{r}, t)\right), \tilde{\eta}_{\phi}(\mathbf{r}, t)=\nabla \cdot\left(\eta_{\phi}(\mathbf{r}, t) \phi^{1 / 2}(\mathbf{r}, t)\right)$, and $\eta_{\phi, \psi}$ satisfies $\left.<\eta_{\phi, \psi}(\mathbf{r}, t) \eta_{\phi, \psi}\left(\mathbf{r}^{\prime}, t^{\prime}\right)\right\rangle=\delta\left(\mathbf{r}-\mathbf{r}^{\prime}\right) \delta\left(t-t^{\prime}\right)$. The second term in Eq.(1) accounts for the TP-TP interactions $\left(\nabla \cdot\left(\psi(\mathbf{r}, t) \int_{\mathbf{r}^{\prime}} \psi\left(\mathbf{r}^{\prime}, t\right) \nabla U\left(\mathbf{r}-\mathbf{r}^{\prime}\right)\right)\right)$ and TP-CC interactions, $\left(\nabla \cdot\left(\psi(\mathbf{r}, t) \int_{\mathbf{r}^{\prime}} \phi\left(\mathbf{r}^{\prime}, t\right) \nabla U\left(\mathbf{r}-\mathbf{r}^{\prime}\right)\right)\right)$. The influence of the CCs on the TP dynamics is reflected in the TP-CC coupling. The third term in Eq.(2) results from cell birth and apoptosis, and the fourth term in Eq.(2) is the non-equilibrium noise. It should be noted that Eq.(1) does not satisfy the fluctuation-dissipation theorem in the tumor growth phase. 
The coupled stochastic integro-differential (SID) equations are difficult to solve analytically. Even numerical solutions in the absence of TPs, are obtained by replacing $\tilde{\eta}_{\phi}(\mathbf{r}, t)$, the last term in Eq. $(2)$, by $\tilde{\eta}_{\phi}(\mathbf{r}, t)=\nabla \cdot\left(\tilde{\eta}_{\phi}(\mathbf{r}, t) \phi_{0}^{1 / 2}\right)$, where $\phi_{0}$ is a constant. Instead of using uncontrolled approximations, we solved the exact coupled SIDs (Eq.(1) and Eq.(2)) numerically (see the methods section for details) in order to calculate the needed correlation functions from which the exponents characterizing the mean square displacement may be derived. For instance, from the time-dependent decay of the correlation function $C_{\psi \psi}(t)=\int d^{3} r C_{\psi \psi}(\mathbf{r}, t)$, we can extract the dynamical exponent, $z$. Previously [14], we had shown that $C_{\psi \psi}(t) \sim t^{1-(2+d) / z}$ ( $d$ is the spatial dimension). The correlation function $C_{\psi \psi}(t) / C_{\psi \psi}(0)$ decays in two stages, depending on $t / \tau$, where $\tau$ is the cell division time (Figure(2a)). For $t / \tau<1$, we find that $C_{\psi \psi}(t) \sim t^{-3 / 7}$ from which we obtain $z=7 / 2$. The MSD $\left(\Delta_{T P}(t)\right)$ exponent for the TPs is related to $z$ as $\Delta_{T P}(t) \sim t^{\beta_{T P}} \sim t^{2 / z}$, which implies $\beta_{T P}=4 / 7$. Similarly, at long $(t / \tau>1)$ times $C_{\psi \psi}(t) \sim t^{-33 / 7}$ from which we deduce that $\Delta_{T P}(t) \sim t^{\alpha_{T P}}$ with $\alpha_{T P}=16 / 7$. Thus, the numerical solution of the exact equations together with the scaling ansatz predict that, in the long time, the TPs undergo hyper-diffusive motion in the presence of CCs.

Simulations: In order to validate the theoretical predictions and decipher the mechanisms of the origin for CC driven hyper-diffusive motion, we simulated a 3D MCS with embedded TPs using an agent-based model [14, 16, 23, 37-39]. The cancer cells, treated as interacting soft deformable spheres, grow with time, and divide into two identical daughter cells upon reaching a critical mitotic radius $\left(R_{m}\right)$. We used, $\tau=\frac{1}{k_{b}}=54,000 \mathrm{~s}$ as the cell division time. The CCs also undergo apoptosis at the rate $k_{a}<<k_{b}$. The sizes and the number of TPs are held constant.

We used a pressure inhibition mechanism to model the observed growth dynamics in solid tumors [14]. Dormancy or the growth phase of the CCs depends on the local microenvironment, which is determined by the pressure on the $i^{\text {th }}$ cell (Figure(1)). Cell division and the placement of the daughter cells stochasticity alter the dynamics of the CCs. More importantly, cell division generates active forces, referred to self-generated active force [23], that persist for a period of time, thus affecting the dynamics of the neighboring cells. To determine the TP dynamics, we model the CC-CC, CC-TP, and TP-TP interactions using two potentials (Gaussian and Hertz) to ensure that the qualitative results are robust (simulation details are in the methods section).

Mean Square Displacement of the TPs $\left(\Delta_{T P}(t)\right)$ : The simulations show that, in the limit $t<\frac{1}{k_{b}}$, the TPs exhibit sub-diffusive behavior with $\Delta_{T P}(t) \sim t^{\beta_{T P}}$ with $\beta_{T P}<1$ (Figure(2b)). Thus, on time scales less than $\tau$ the TPs exhibit sub-diffusive behavior, as predicted theoretically (2a)). We also calculated $\Delta_{T P}(t)=\left\langle[\mathbf{r}(t)-\mathbf{r}(0)]^{2}\right\rangle$, by averaging over $\approx 2,000$ trajectories, for the Gaussian (Figure $(2 b)$ ) and Hertz (Figure S3a in the SI) potentials. Similar behavior is found in both the cases. The modest increase in the MSD at short times is seen as a near plateau in $\Delta_{T P}(t)$ as a function of $t($ Figure $(2 \mathrm{~b}))$. The duration of the plateau (Figure $\left.(2 \mathrm{~b})\right)$ increases as the cell cycle time increases. Although jamming behavior predicted theoretically is consistent with the simulations the numerical values of $\beta_{T P}$ differ (compare Figure(2a) and (2b)). The dynamics in the $t / \tau<1$ limit depends on the details of the model.

In the $t>\frac{1}{k_{b}}$ limit, theory and simulations predict hyper-diffusive dynamics for the TPs, $\Delta_{T P} \sim t^{\alpha_{T P}}$ with $\alpha_{T P} \approx 2.3$. Variations in $k_{b}=\tau^{-1}$ do not change the value of $\alpha_{T P}$ (Figure(2b)). It merely changes the coefficients of the linear term and the value of $\frac{2 k_{b}}{k_{a}}$. Therefore, $\alpha_{T P}$ is independent of the cell cycle time in the long time limit. Simulation results are in excellent agreement with the theoretical predictions. For the Gaussian potential, we obtain $\alpha_{T P} \approx 2.3$ (see the inset in Figure $(2 \mathrm{~b})$ ) for $0.5 \tau, \tau$ and $2 \tau$. Thus, $\alpha_{T P}$ is independent of cell cycle time, as argued above. Note that TP-TP interactions play an insignificant role in the dynamics of TPs or the CCs (Figure S4 in the SI). They merely alter the amplitude of $\Delta_{T P}$ in the intermediate time. They do not affect the long time dynamics. We conclude that the long-time behavior is universal, and is impervious to the details of the interaction but is determined by cell division and apoptosis times.

Impact of TP on CC dynamics: Interestingly, the time-dependent changes in $\Delta_{C C}(t)$ are not significantly affected by the TPs. Figure(3) shows that changing the tracer size affects only the amplitude of the $\Delta_{C C}$ in the intermediate time without altering the $\alpha_{C C}$ values (see also figure S5 in the SI). This is because the TP size only changes the nature of the short-range interactions without introducing any new length scale. Since, $\alpha_{C C}$ is a consequence of the long-range spatial and temporal correlations that emerge because of the rates cell division and apoptosis, it is independent of the tracer size (see the details in the SI).

In the absence of the TPs, birth and apoptosis determine the CC dynamics in the long time regime, yielding $\alpha_{C C}=1.33[14,15]$. When the TPs are present, the CCs continue to exhibit super-diffusive motion with a modest increase in $\alpha_{C C}$ (Table II in the SI). The origin of the predicted super-diffusive behavior in the CCs is related to the long range spatial correlation that arises due the SGAF that is related to cell division. This is reflected in the CC pair-correlation, $g(r)=\frac{V}{4 \pi r^{2} N^{2}} \sum_{i=1}^{N} \sum_{j \neq i}^{N} \delta\left(r-\left|\mathbf{r}_{i}-\mathbf{r}_{j}\right|\right) \sim r^{-0.5}$, in the presence and absence of TPs at $t \approx 8 \tau$ (Figure(4a)). The dynamically-induced long-range CC correlations is independent of the TPs, thus explaining the insignificant effect of the TPs on the CC dynamics. 


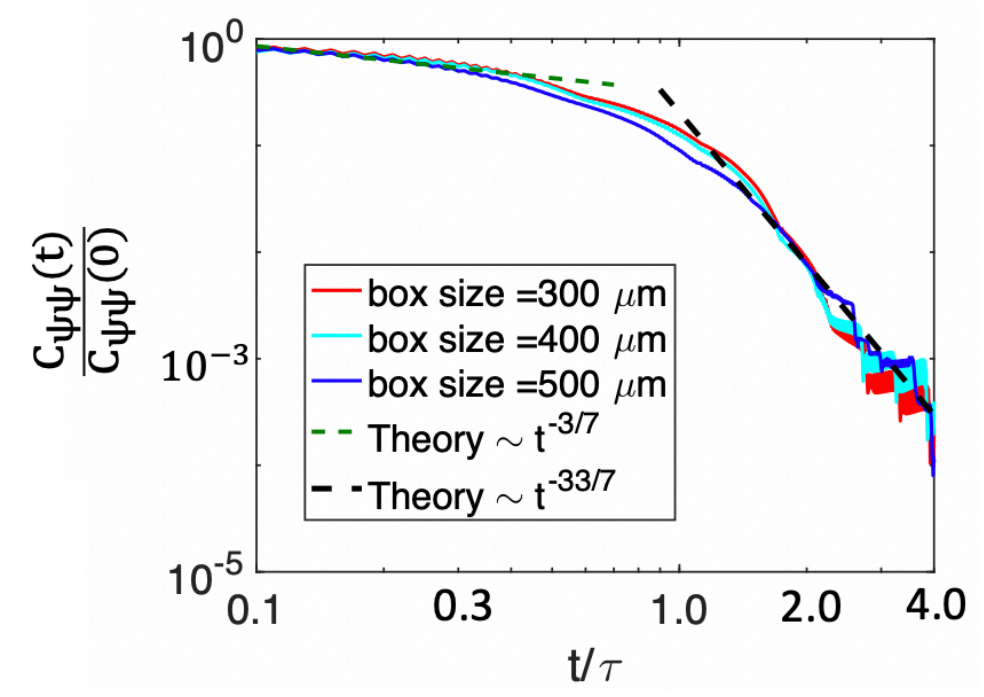

(a)

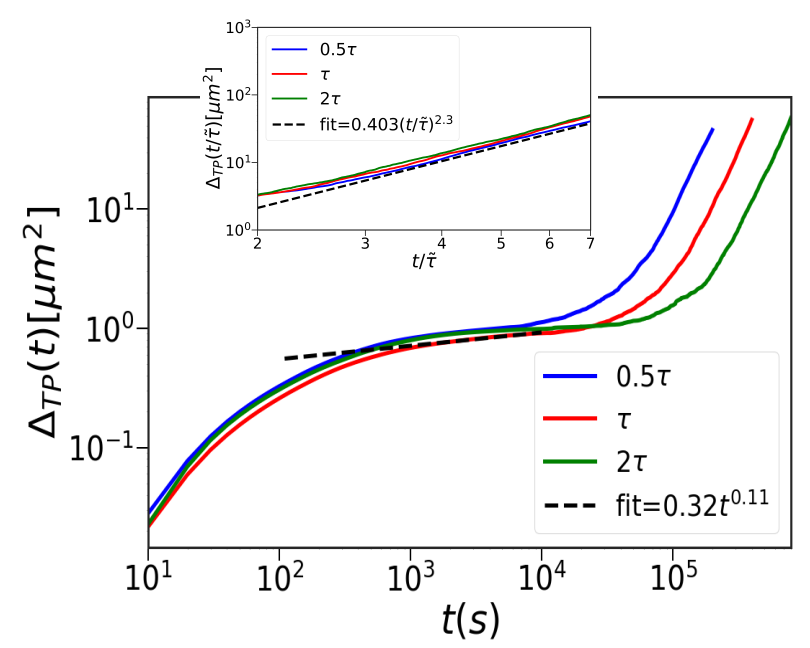

(b)

Figure 2: (a) The normalized time-dependent density correlation function plot for the TPs. The cell cycle time is $\tau=54,000 \mathrm{~s}$. The integrated time $(t \approx 4 \tau)$ is long enough to obtain accurate exponents from the power law decay for both short $(t<\tau)$ and long times $(t>\tau)$. The plots for three different box sizes confirm that the values of the exponents do not depend on the system size. (b) MSD of the TPs $\left(\Delta_{T P}\right)$ using the Gaussian potential. The curves are for 3 cell cycle times (blue $(0.5 \tau)$, red $(\tau)$, and green $(2 \tau)$. Time to reach the hyper-diffusive behavior, which is preceded by a jamming regime $\left(\Delta_{T P} \sim t^{\beta_{T P}}, \beta_{T P}=0.11\right.$, shown in black dashed line), increases with $\tau$. The inset focusses on the hyper-diffusive regime $\left(\frac{t}{\tilde{\tau}}>1\right) . \tilde{\tau}$ is the cell cycle time for the respective curves. Time is scaled by $\frac{1}{\tilde{\tau}} . \Delta_{T P} \sim t^{\alpha_{T P}}, \alpha_{T P}=2.3$ (dashed black line).

Mechanistic Origin: To explain the finding that, $\alpha_{T P}>\alpha_{C C}$, we calculated the force-force autocorrelation function, $\mathrm{FFA}=\left\langle\mathbf{F}\left(t+t_{d}\right) \cdot \mathbf{F}(t)\right\rangle_{t}$. Here, $\langle\ldots\rangle_{t}$ is the time average and $t_{d}$ is the duration of the delay time. Since, the TPs (CCs) exhibit hyper-diffusion (super-diffusion), we expect that the FFA of TPs should decay slower relative to the CCs. This is confirmed in Figure(4b), which shows that the FFA for the TPs (CCs) decays as $t_{d}^{-0.7}\left(t_{d}^{-1.2}\right)$. Thus, the TP motion is significantly more persistent than the CCs, which explains the hyper-diffusive nature of the TPs.

A mechanistic explanation for $\alpha_{T P}>\alpha_{C C}$ can be derived from the simulations. To illustrate the difference between the dynamics of the TPs and CCs, we calculated the angle $(\theta)$ between two consecutive time steps in a trajectory. We define $\cos (\theta(t, \delta t))=\frac{\delta \mathbf{r}(t+\delta t) \cdot \delta \mathbf{r}(t)}{|\delta \mathbf{r}(t+\delta t)| \mid \delta \mathbf{r}(t)) \mid}$, where $\delta \mathbf{r}(t)=\mathbf{r}(t+\delta t)-\mathbf{r}(t)$. Figure(5a) shows the ensemble and time averaged $\cos \theta$ distribution for $\frac{\delta t}{\tau}=1$. If the motion of the CCs and TPs were diffusive, the distribution of $\cos \theta$ would be uniformly distributed from -1 to 1 . However, Figure(5a) shows that $P(\cos \theta)$ is skewed towards unity implying that the motion of both the TPs and CCs are persistent. Interestingly, the skewness is more pronounced for the 


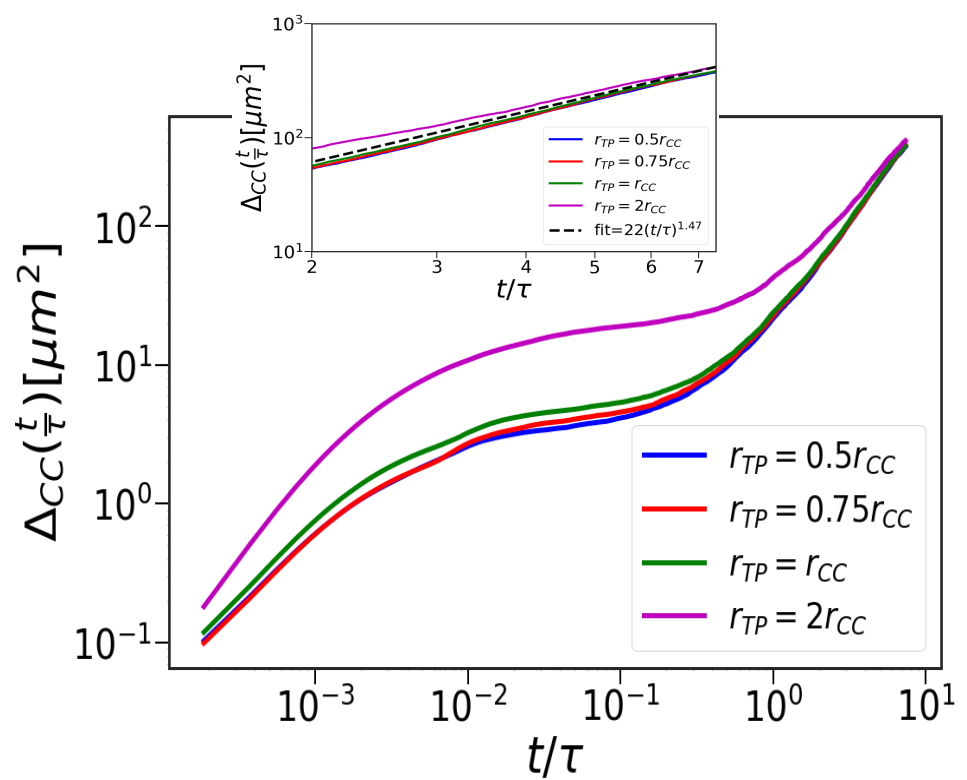

Figure 3: CC dynamics in the presence of the TPs. $\Delta_{C C}$ using the Hertz potential. The curves are for different TP radius ( magenta $-r_{T P}=2 r_{C C}$, green $-r_{T P}=r_{C C}$, and red $-r_{T P}=0.75 r_{C C}$, and blue $-r_{T P}=0.5 r_{C C}$, where $r_{C C}=4.5 \mu m$ is the average cell radius. Inset shows $\Delta_{C C}$ for the four curves, focusing on the super-diffusive regime. The black dashed line is drawn with $\alpha_{C C}=1.47$.

TPs compared to the CCs (Figure(5a)). The enhanced persistence of TP motion due to SGAF is also reflected in the FFA(t) displayed in Figure(4b). The slower decay of FFA(t) (Figure(4b)) in the TPs compared to the CCs is neglected in the distribution of $P[\cos (\theta)]$, shown in Figure(5a). During each cell division, the motion of the CCs is randomized, and hence the persistence is small compared to TPs. This explains the hyper-diffusive (super-diffusive) for the TPs (CCs) and thus $\alpha_{T P}>\alpha_{C C}$ in the long time limit (see Figure(5a) which shows TPs movement is much more persistent than $\mathrm{CCs}$ ).

Monotonic decrease of pressure from the core to periphery of the MCS: To assess the effectiveness of the TPs as sensors of the local microenvironment, we calculated the radial pressure (Eq.(10) in the methods section) profile for both CCs and TPs at $t=7.5 \tau$. In order to distinguish between the local pressure due to the TPs and CCs, we calculated pressure experienced just by the TPs. Finally, we computed pressure in a system consisting of only the CCs. By comparing the results from different simulations, we can unambiguously establish that TPs are excellent reporters of the local stresses within a single tumor.

From the results shown in Figure(5b), we draw the following conclusions. (i) All three curves qualitatively capture the pressure profile found in the experiment [32]. From the results in Figure(5b) several important conclusions that are relevant to experiments may be drawn. Pressure decreases roughly by a factor of four, as the distance, $r$, from the center of the tumor increases. The pressure is almost constant in the core, with a decrease that can be fit using the logistic function, as the boundary of the tumor is reached (Figure(5b)). The experimental data, shown in the inset in Figure(5b), was fit using a power law $\left(\sim r^{-\beta}\right.$ with $\left.\beta \approx 0.2\right)$ (see Figure(4b) in the experiment [32]). Both the fits account for the data with the important point being that the stress is non-uniform across the solid tumor.

(ii) The experiments used reduction in volume of the polyacrylamide microbeads upon external compression $(=$ $5 \mathrm{KPa}$ ) as local pressure sensors in colon carcinoma cells. Although our simulations qualitatively reproduce the experimental pressure profile, the magnitude of the stress in the simulations is about three orders of magnitude less relative to experiments. The most likely reason is that in experiments high stress is uniformly applied by compressing the spherical tumor. In contrast, in our simulations pressure arises explicitly due to SGAF arising from an interplay of systematic forces, cell division, as well as mechanical feedback. The simulated MCS is not intended to mimic the characteristics of the colon carcinoma cells studied in the experiment [32]. It should also be noted that the magnitude of the pressure is also dependent on the tissue type. For example, a recent experiment [33], using fluorescently labeled water-soluble peptide-based microtubes, showed that the measured pressure $\approx 0.4 K P a$. The qualitative agreement between experiments and simulations (Figure $(5 \mathrm{~b})$ ) suggests that the dynamical rearrangement due to cell division and apoptosis and mechanical feedback controls the observed radial variations in the pressure profiles.

(iii) The higher value of pressure in the core of the MCS as the MCS grows is a consequence of jamming, which increase the local density. Because of the rapid cell division rate, the pressure experienced by cells in the interior is high (Figures 5b, 5c and 5d). As a result, the local stresses in the core of the MCS do not have enough time to 


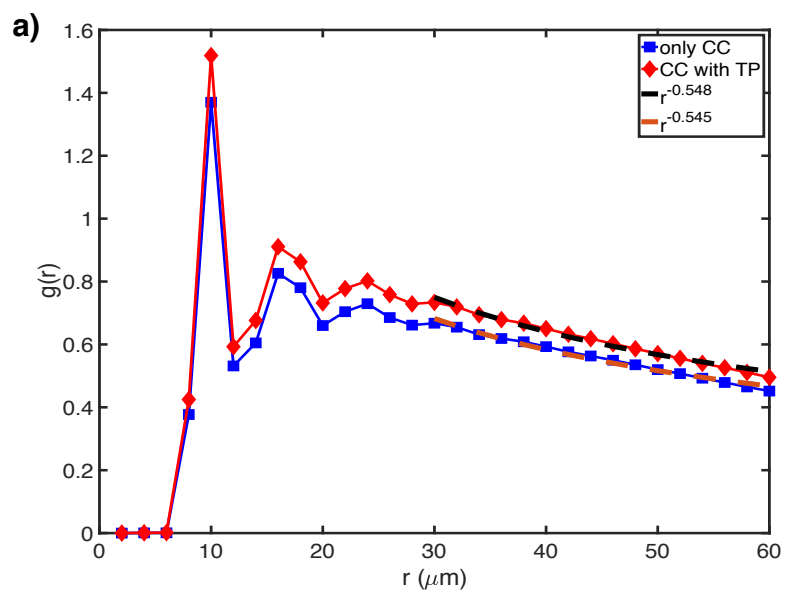

b)

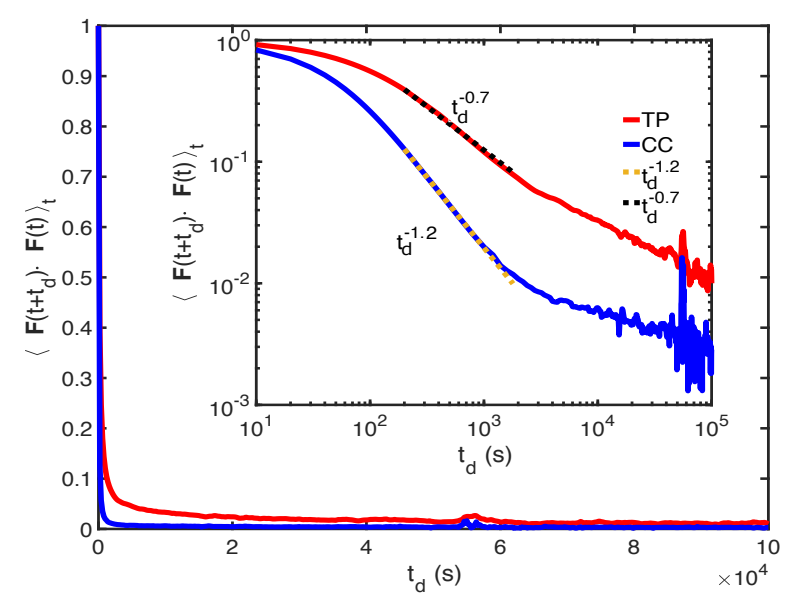

Figure 4: (a) CC correlation function, $(g(r))$, as a function of inter-cellular distance. Red (blue) curve shows $g(r)$ in presence (absence) of TPs. The two dashed lines (black and orange) are power law fits to $g(r)$ in the large $r$ limit. (b) Force force autocorrelation (FFA), as a function of delay time $\left(t_{d}\right)$. The red (blue) curve shows the FFA for TPs (CCs). Inset shows FFA on log-log scale. The black (yellow) dashed line is a power law fit with exponent of -0.7 (-1.2).

relax. The higher core density implies that pressure in the interior increases and exceeds $p_{c}$, which further inhibits proliferation, thus deceasing the number of cell divisions. The reduction in the number of cell divisions, which is a mechanism by which stress relaxation occurs, leads to increase in pressure at $r<R_{0}$ (Figures (5b, $5 \mathrm{c}$ and $5 \mathrm{~d}$ )). As $r$ increases, the jamming effects diminish, local stress relaxation is faster resulting in the pressure on the cells being less than $p_{c}$. Consequently, the CCs proliferate, resulting in a decrease in self-generated stress, and consequently, a decreases in the magnitude of the pressure (Figures (5b, 5c and 5d)).

(iv) Most importantly, the CC pressure profiles are unaltered even in the presence of the TPs (compare blue circles and greenish-yellow squares in Figure 5b, which shows that the latter does faithfully report the microenvironment of the CCs. In addition to the radial profile, we also show that the CC stress distribution is unaltered with and without TPs in Figure 5e. The plot in Figure 5f establishes that the stress distribution on the TPs roughly approximates the stress distribution on the CCs, implying that TPs can serve as reporters of not only the radial profile (Figure 5b) but also the CC stress distribution.

The simulations also provide plausible reasons for the decrease in the pressure from the center to the boundary of the tumor. Pressure on the $i^{\text {th }}$ cell located at a distance $r$ from the tumor center not only, depends on the total force exerted by the neighboring cells but also is controlled by cell division, which is a dynamic process. As the tumor evolves through cell division, the local density $\phi(\mathbf{r})$ of the cells at small $\mathbf{r}$ increases. More importantly, cell division induces local stresses [40], which would relax on time scales that depend on $\phi(\mathbf{r})$. Thus, cell division, jamming due to an increase in $\phi(\mathbf{r})$, and stress relaxation times are intimately related. The smaller $\phi(\mathbf{r})$ is faster in the stress 


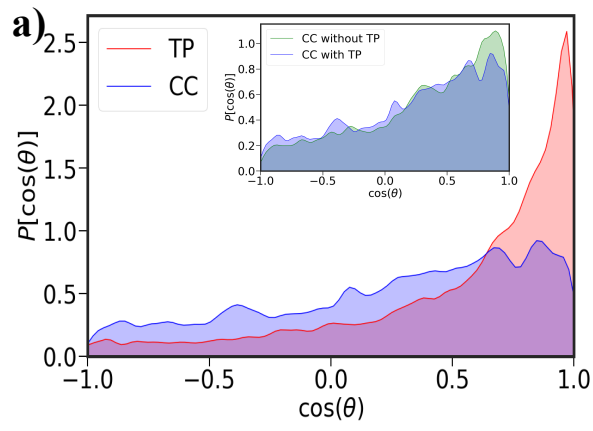

c)

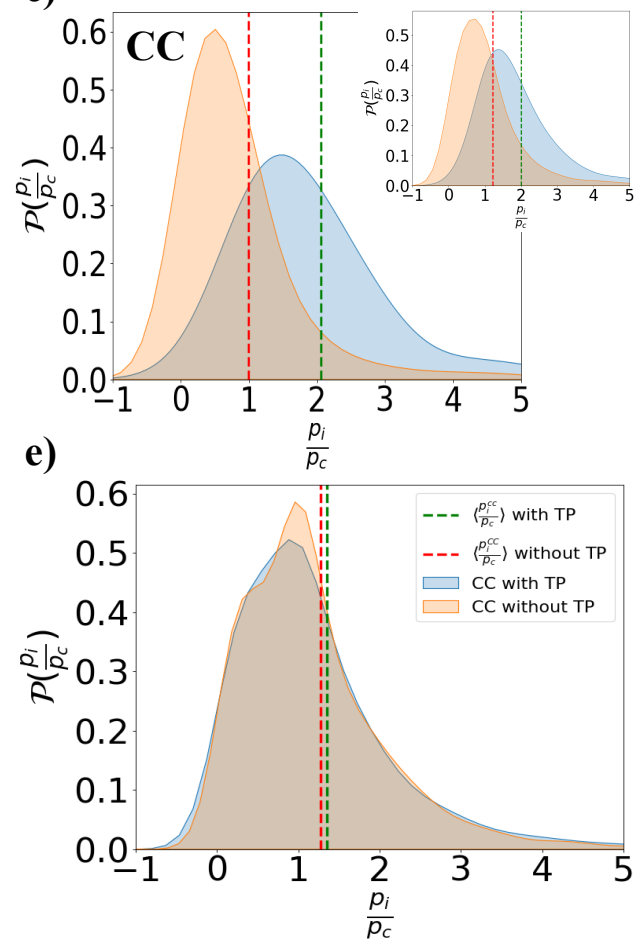

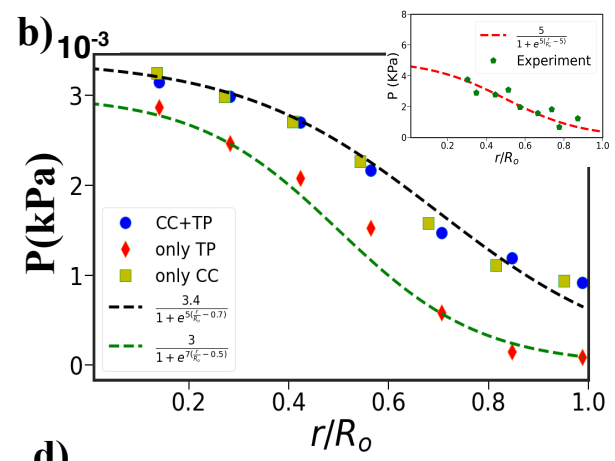

d)
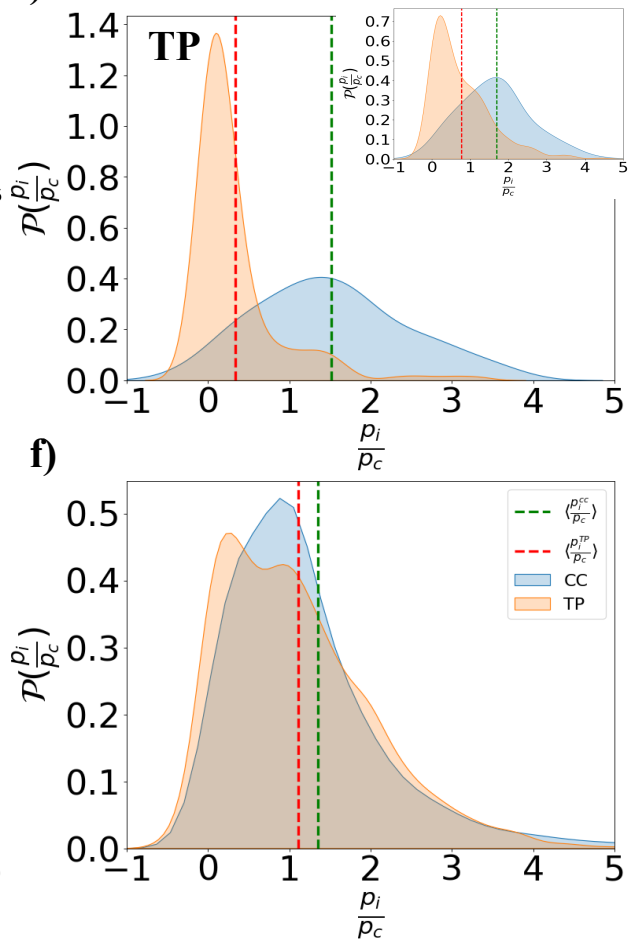

Figure 5: (a) Distribution of $\cos (\theta)$ for the TPs and CCs. $\theta$ is the angle between two consecutive steps along a trajectory. The red (blue) plot is for the TPs (CCs). The distribution is skewed to $\cos (\theta)>0$, indicative of persistent motion. Extent of skewness is greater for TPs than CCs. The inset shows the $P[\cos (\theta)]$ of CCs with and without TPs. (b)Pressure as a function of the radial distance ( $r$ scaled by $R_{0}$ : tumor radius $\approx 100 \mu m$ ) from tumor center. The blue circles correspond to local pressure measured using both CCs and TPs. The red data points is local pressure measured using just the TPs using simulations containing both the CCs and TPs. The greenish yellow squares give the local pressure obtained in simulations with only the CCs. The black and green dashed lines is logistic fit. The inset shows the radial pressure profile in experiments (green data points). The red dashed lines are logistic fits. Note that the magnitude of pressure measured in simulations and experiments are in qualitative agreement. (c) Pressure probability distribution, $\mathcal{P}\left(\frac{p_{i}}{p_{c}}\right)$ for CCs. In the main figure, orange (blue) distributions correspond to stress distribution on CCs whose distance is greater (less) than $80 \mu m(20 \mu m)$. Similarly in the inset, orange (blue) distributions correspond to stress distribution on CCs whose distance is greater (less) than $60 \mu m$ $(30 \mu \mathrm{m})$. (d) Same as (c), except the distributions correspond to TPs. (e) The blue (orange) distribution corresponds to the stress distributions on the CCs in the presence (absence) of the TPs. (f) The blue (orange) distribution are the stress distributions on all the CCs (TPs) in the spheroid.

relaxation time, and the smaller is the value of the pressure. It is clear that near the periphery of the tumor $\phi(\mathbf{r})$ is smaller than at the core, which explains the origin of the pressure profile in Figure 5b.

Conclusions: We used simulations and theory to elucidate the dynamics of inert tracer particles that are embedded in an evolving multicellular spheroid (MCS). Surprisingly, theory and simulations show that for $k_{b} t>1$ the TPs exhibit hyper-diffusive behavior, which does not depend on the details of interaction between the cells. In contrast, the exponent, $\alpha_{C C}$, characterizing the long-time behavior of $\Delta_{C C}(t)$ is nearly the same with or without the TP, and is also independent of the probe radius. This suggests that TPs may be used to probe the behavior of cancer cells 
within a solid tumor, which cannot be established unambiguously experiments alone.

The most direct connections to experiments were made by showing that the simulated and measured stress profiles are qualitatively similar. This is unexpected because the protocol used in experiments (response to an isotropically applied stress to a spheroid) is completely different in the simulations in which stresses within the MCS are selfgenerated. The higher stress values in the interior of the spheroid imply that proliferation is suppressed in the core. The most important finding is that the CC pressure profiles are virtually identical independent of the presence or absence if TPs (Figure(5(b)), which shows that the TPs are reporters of the CC microenvironment in a tumor.

An explanation for the radial dependence of local stresses in Figure(5(b) is related to the non-uniform proliferation of cells in the MCS. In the models, we used mechanical feedback to control the growth of the MCS. This implies that if the local stresses exceed a critical value cells become dormant, and cannot growth until the neighboring cells rearrange to decrease the pressure on a labeled cell. However, as the MCS evolves, the pressure on cells that are in the interior could exceed a critical value $\left(p_{c}\right)$ due to jamming. In contrast, the pressure on cells near the MCS boundary is typically below $p_{c}$, which results in enhanced proliferation. It is the non-uniformity in the cell division rates due to mechanical feedback that not only is the cause of the radial variation in pressure in the MCS but also gives rise to the complicated radial dependent dynamics (glass-like slow dynamics in the interior to super diffusion near the periphery) [16]. The implication is that the effective diffusion constants of CCs as well as the TPs also vary with distance from the center of the MCS increases.

\section{Methods:}

Numerical solution of the SIDs (Eq.(1) and Eq.(2)): We numerically integrated Eq.(1) and Eq.(2) by discretizing space in a cubic box with periodic boundary conditions. The size of the $3 \mathrm{D}$ box with $L_{x}=L_{y}=L_{z}$ is varied from 300 to $500 \mu m$ to ensure that the results do not depend on the system size. The 3D box was divided into grids, each with volume $\delta x \times \delta y \times \delta z$ with $\delta x=\delta y=\delta z=10 \mu \mathrm{m}$. The parameters used in the numerical integration scheme are shown in Table I in the SI. Note that the precise shape of the box does not affect the numerical solutions nor does the value of $d x$ as long the solutions converge without numerical instability.

Simulations: In order to test the theoretical predictions, and provide mechanistic insights underlying the unusual dynamics of the TPs in the MCS, we simulated a 3D tumor spheroid with embedded TPs. Following previous studies, we used an agent based model $[14,37]$ for the tumor spheroid. The cells are treated as deformable spheres. The size of the CCs increase with time, and divide into two identical cells upon reaching a critical mitotic radius $\left(R_{m}\right)$. The mean cell cycle time, $\tau=54,000 \mathrm{~s}$, which is a realistic value for fibroblast cells. The CCs can also undergo apoptosis with rate $k_{a}$. The birth rate, $k_{b}=\frac{1}{\tau}$ is large compared to the death rate $\left(\frac{k_{b}}{k_{a}}=20\right)$, which mimics the growth of the MCS. As in the theory, the TPs are inert, and their sizes and the number are constant in the simulations. We use two different potentials for the CC-CC, CC-TP and TP-TP interactions.

Hertz potential: The form of the Hertz forces between the CCs is the same as in the previous studies [14, 16, 37, $41,42]$. The physical properties of the CC, such as the radius, elastic modulus, membrane receptor, and E-cadherin concentration characterize the strength of the inter-cellular interactions. The elastic forces between two spheres with radii $R_{i}$ and $R_{j}$, is given by,

$$
F_{i j}^{e l}=\frac{h_{i j}^{\frac{3}{2}}}{\frac{3}{4}\left(\frac{1-\nu_{i}^{2}}{E_{i}}+\frac{1-\nu_{j}^{2}}{E_{j}}\right)\left(\sqrt{\frac{1}{R_{i}}+\frac{1}{R_{j}}}\right)},
$$

where $E_{i}$ and $\nu_{i}$ are, respectively, the elastic modulus and Poisson ratio of the $i^{\text {th }}$ cell. We assume that $E_{i}$ and $\nu_{i}$ are independent of $i$. Since the CCs or the TPs are deformable, the elastic force depends on the overlap, $h_{i j}$, between two cells. The adhesive force, $F_{i j}^{a d}$, between the CCs is proportional to the area of contact $\left(A_{i j}\right)$ [43], and is calculated using, [41],

$$
F_{i j}^{a d}=A_{i j} f^{a d} \Lambda_{0}
$$

where $\Lambda_{0}$ is unity in the present study.

Repulsive and adhesive forces in Eqs.(3) and (4) act along the unit vector $\vec{n}_{i j}$ connecting the centers of cells $j$ and $i$. Therefore, the net force on cell $i\left(\vec{F}_{i}^{H}\right)$ is given by the sum over the nearest neighbors [NN $\left.(\mathrm{i})\right]$,

$$
\vec{F}_{i}^{H}=\Sigma_{j \in N N(i)}\left(F_{i j}^{e l}-F_{i j}^{a d}\right) \vec{n}_{i j}
$$


To model the TP-TP and TP-CC interactions, we assume that the TPs are CC-like objects [32]. Therefore, CC-TP and TP-TP interactions are the same as CC-CC interactions. The parameters used in the simulations using the Hertz potential (Eqs.(3) and (4)) are given in Table I in the SI. We initiated the simulations with 100 TPs and 100 CCs. The coordinates of the CCs and TPs were sampled using a normal distribution with zero mean, and standard deviation $50 \mu \mathrm{m}$. The initial radii of the CCs and TPs were sampled from a normal distribution with mean $4.5 \mu \mathrm{m}$, and a dispersion of $0.5 \mu \mathrm{m}$.

Gaussian potential: In the theoretical treatment, we assumed that the CC-CC interaction is given by a sum of Gaussian terms (Eq. (S2) in the SI). For this potential, the force $\mathbf{F}_{i j}^{G}$ on cell $i$, exerted by cell $j$, is,

$$
\mathbf{F}_{i j}^{G}=\frac{1}{(2 \pi)^{3 / 2}}\left[\frac{\nu e^{\frac{-r^{2}}{2 \lambda^{2}}}}{\lambda^{5}}-\frac{\kappa e^{\frac{-r^{2}}{2 \sigma^{2}}}}{\sigma^{5}}\right] \mathbf{r}
$$

where $\mathbf{r}$ is $\mathbf{r}(i)-\mathbf{r}(j)$. We write $\lambda$ and $\sigma$ as $\lambda=\widetilde{\lambda}\left(R_{i}+R_{j}\right)$ and $\sigma=\widetilde{\sigma}\left(R_{i}+R_{j}\right)$, as the ranges of interactions corresponding to the repulsive and attractive interactions, respectively. In our simulations, the CCs grow and divide, their radii change in time, and therefore $\lambda$ and $\sigma$ also change with time. However, since these interactions are shortranged, we assume that they are constant in order to be consistent with the assumption in the theory. We fixed $\lambda=\widetilde{\lambda}\left(2 R_{d}\right)$ and $\sigma=\widetilde{\sigma}\left(2 R_{d}\right)$, where $R_{d}(\approx 4 \mu \mathrm{m})$ is the size of a daughter cell (introduced in the next section). For simplicity, we write the force as $\mathbf{F}_{i j}^{G}=\left[\frac{\widetilde{\nu} e^{\frac{-r^{2}}{2 \lambda^{2}}} \lambda^{2}}{\frac{\widetilde{\kappa}}{\lambda^{2}}{\frac{-r^{2}}{2 \sigma^{2}}}_{\sigma^{2}}}\right] \mathbf{r}$, where $\widetilde{\nu}=\frac{1}{(2 \pi)^{3 / 2}} \frac{\nu}{\lambda^{3}}$ and $\widetilde{\kappa}=\frac{1}{(2 \pi)^{3 / 2}} \frac{\kappa}{\sigma^{3}}$. In the simulations, we fixed $\widetilde{\nu}=0.03, \widetilde{\lambda}=0.28, \widetilde{\kappa}=0.003$ and $\widetilde{\sigma}=0.4$.

Cell division, Dormancy and Apoptosis: The CCs are either dormant or in the growth phase depending on the microenvironment, which is assessed using the value of the local pressure. The pressure on cell $i\left(p_{i}\right)$ due to $N N(i)$ neighboring cells is calculated using the Irving-Kirkwood equation,

$$
p_{i}=\frac{1}{3 V_{i}} \Sigma_{j \epsilon N N(i)} \mathbf{F}_{i j} \cdot d \mathbf{r}_{i j},
$$

where $\mathbf{F}_{i j}$ is the force on the $i^{t h}$ cell due to $j^{t h}$ cell and $d \mathbf{r}_{i j}=\mathbf{r}_{i}-\mathbf{r}_{j}$. The volume of the $i^{\text {th }}$ cell $\left(V_{i}\right)$ is $\frac{4}{3} \pi R_{i}^{3}$, where $R_{i}$ is the cell radius. If $p_{i}$ exceeds a pre-assigned critical limit $p_{c}(=1.7 \mathrm{~Pa})$ the $\mathrm{CC}$ enters a dormant phase. The dormancy criterion serves as a source of mechanical feedback, which limits the growth of the tumor spheroid [44-48]. The volume of a growing cell increases at a constant rate, $r_{V}$. The cell radius is updated from a Gaussian distribution with the mean rate $\dot{R}=\left(4 \pi R^{2}\right)^{-1} r_{V}$. Over the cell cycle time $\tau$, the cell radius increases as,

$$
r_{V}=\frac{2 \pi\left(R_{m}\right)^{3}}{3 \tau}
$$

where $R_{m}$ is the mitotic radius. A cell divides once it grows to the fixed mitotic radius. To ensure volume conservation, upon cell division, we use $R_{d}=R_{m} 2^{-1 / 3}$ as the radius of the daughter cells. The resulting daughter cells are placed at a center-to-center distance $d=2 R_{m}\left(1-2^{-1 / 3}\right)$ (Figure(1)). The direction of the new cell location is chosen randomly from a uniform distribution on a unit sphere. At times $\sim k_{a}^{-1}$, a randomly chosen cell undergoes apoptosis.

Equation of Motion: The equation of motion governing the dynamics of the TP and CCs is taken to be,

$$
\dot{\vec{r}}_{i}=\frac{\vec{F}_{i}}{\gamma_{i}}
$$

where $\dot{\vec{r}}_{i}$ is the velocity of $i^{\text {th }} \mathrm{CC}$ or TP, $\vec{F}_{i}$ is the force on $i^{t h} \mathrm{CC} / \mathrm{TP}$ (see equation 5 and 6 ), and $\gamma_{i}$ is the damping term. The details including the rationale for neglecting the random forces and inertial forces, are given elsewhere [14].

Radial dependence of pressure: We calculated the dependence of pressure $P(r)$ at a distance $r$ from the center of the tumor using,

$$
P(r)=\frac{1}{n(r)} \sum_{i=1}^{n(r)} p_{i}
$$


where $p_{i}$ is the pressure (Eq.(7)) on the $i^{t h}$ cell located between $r$ and $r+d r$, and $n(r)$ is the number of cells in the same annulus. It is worth remembering that if the MCS is at equilibrium, $P(r)$ would be independent of $r$. Because in the simulations, cell proliferation is enhanced compared to the core of the MCS, there is more rapid relaxation of the stress at the MCS boundary, which result in reduced pressure.

In order to show that cell division is more likely to occur away from the core than in the interior of the MCS, we also calculated distribution $\left(P\left(p_{i}(r)\right)\right)$ of pressure experienced by cells in the annulus between $r$ and $r+d r$. We calculated $p_{i}(r)$ using the equation similar to Eq.(10) except in the $n(r)$ is the number of cells in the spherical volume between $r$ and $r+d r$.

Acknowledgements: We thank Xin Li, Abdul N. Malmi-Kakkada, Mauro Mugnai, Hung Nguyen, Rytota Takaki and Davin Jeong for useful discussions and comments on the manuscript. This work is supported by the National Science Foundation (PHY 17-08128), and the Collie-Welch Chair through the Welch Foundation (F-0019).

[1] Friedl, P.; Gilmour, D. Collective cell migration in morphogenesis, regeneration and cancer. Nat. Rev. Mol. Cell Biol. 2009, 10, 445-457.

[2] Shaebani, M.; Wysocki, A.; Winkler, R.; Gompper, G.; Rieger, H. Computational models for active matter. Nat. Rev. Phys. 2020, 2, 181-199.

[3] Kumar, S.; Weaver, V. M. Mechanics, malignancy, and metastasis: the force journey of a tumor cell. Cancer Metastasis Rev. 2009, 28, 113-127.

[4] Desoize, B.; Jardillier, J.-C. Multicellular resistance: a paradigm for clinical resistance? Crit. Rev. Oncol. Hemat. 2000, 36, 193-207.

[5] Angelini, T. E.; Hannezo, E.; Trepat, X.; Marquez, M.; Fredberg, J. J.; Weitz, D. A. Glass-like dynamics of collective cell migration. Proc. Natl. Acad. Sci. 2011, 108, 4714-4719.

[6] Walenta, S.; Doetsch, J.; Mueller-Klieser, W.; Kunz-Schughart, L. A. Metabolic imaging in multicellular spheroids of oncogene-transfected fibroblasts. J Histochem Cytochem. 2000, 48, 509-522.

[7] Laurent, J.; Frongia, C.; Cazales, M.; Mondesert, O.; Ducommun, B.; Lobjois, V. Multicellular tumor spheroid models to explore cell cycle checkpoints in 3D. BMC cancer 2013, 13, 73-84.

[8] Valencia, A. M. J.; Wu, P.-H.; Yogurtcu, O. N.; Rao, P.; DiGiacomo, J.; Godet, I.; He, L.; Lee, M.-H.; Gilkes, D.; Sun, S. X., et al. Collective cancer cell invasion induced by coordinated contractile stresses. Oncotarget 2015, 6, 43438-43451.

[9] Han, Y. L. et al. Cell swelling, softening and invasion in a three-dimensional breast cancer model. Nature Physics 2020, $16,101-108$.

[10] Richards, R.; Mason, D.; Levy, R.; Bearon, R.; See, V. 4D imaging and analysis of multicellular tumour spheroid cell migration and invasion. bioRxiv 2018, 443648-443663.

[11] Martino, N.; Kwok, S. J.; Liapis, A. C.; Forward, S.; Jang, H.; Kim, H.-M.; Wu, S. J.; Wu, J.; Dannenberg, P. H.; Jang, S.-J., et al. Wavelength-encoded laser particles for massively multiplexed cell tagging. Nat. Photonics 2019, 13, $720-727$.

[12] Han, Y. L.; Pegoraro, A. F.; Li, H.; Li, K.; Yuan, Y.; Xu, G.; Gu, Z.; Sun, J.; Hao, Y.; Gupta, S. K., et al. Cell swelling, softening and invasion in a three-dimensional breast cancer model. Nat. Phys. 2019, 1-8.

[13] Palamidessi, A.; Malinverno, C.; Frittoli, E.; Corallino, S.; Barbieri, E.; Sigismund, S.; Beznoussenko, G. V.; Martini, E.; Garre, M.; Ferrara, I., et al. Unjamming overcomes kinetic and proliferation arrest in terminally differentiated cells and promotes collective motility of carcinoma. Nat. mater. 2019, 1-12.

[14] Malmi-Kakkada, A. N.; Li, X.; Samanta, H. S.; Sinha, S.; Thirumalai, D. Cell Growth Rate Dictates the Onset of Glass to Fluidlike Transition and Long Time Superdiffusion in an Evolving Cell Colony. Phys. Rev. X 2018, 8, 021025-021045.

[15] Samanta, H. S.; Thirumalai, D. Origin of superdiffusive behavior in a class of nonequilibrium systems. Phys. Rev. E 2019, 99, 032401-032408.

[16] Sinha, S.; Malmi-Kakkada, A. N.; Li, X.; Samanta, H. S.; Thirumalai, D. Spatially heterogeneous dynamics of cells in a growing tumor spheroid: comparison between theory and experiments. Soft Matter 2020, 16, 5294-5304.

[17] Bi, D.; Lopez, J. H.; Schwarz, J. M.; Manning, M. L. A density-independent rigidity transition in biological tissues. Nature Physics 2015, 11, 1074-1079.

[18] Bi, D.; Yang, X.; Marchetti, M. C.; Manning, M. L. Motility-Driven Glass and Jamming Transitions in Biological Tissues. Phys. Rev. X 2016, 6, 021011-021023.

[19] Park, J.-A. et al. Unjamming and cell shape in the asthmatic airway epithelium. Nature Materials 2015, 14, 1040-1048.

[20] Marchetti, M. C.; Joanny, J.-F.; Ramaswamy, S.; Liverpool, T. B.; Prost, J.; Rao, M.; Simha, R. A. Hydrodynamics of soft active matter. Rev. Mod. Phys. 2013, 85, 1143-1189.

[21] Bechinger, C.; Di Leonardo, R.; Löwen, H.; Reichhardt, C.; Volpe, G.; Volpe, G. Active particles in complex and crowded environments. Rev. Mod. Phys. 2016, 88, 045006-045055.

[22] Nandi, S. K.; Mandal, R.; Bhuyan, P. J.; Dasgupta, C.; Rao, M.; Gov, N. S. A random first-order transition theory for an active glass. Proc. Natl. Acad. Sci. 2018, 115, 7688-7693.

[23] Sinha, S.; Thirumalai, D. Self-generated persistent random forces drive phase separation in growing tumors. J. Chem. Phys. 2020, 153, 201101-201106. 
[24] Boucher, Y.; Baxter, L. T.; Jain, R. K. Interstitial Pressure Gradients in Tissue-isolated and Subcutaneous Tumors: Implications for Therapy. Cancer Research 1990, 50, 4478-4484.

[25] Helmlinger, G.; Netti, P. A.; Lichtenbeld, H. C.; Melder, R. J.; Jain, R. K. Solid stress inhibits the growth of multicellular tumor spheroids. Nature Biotechnology 1997, 15, 778-783.

[26] Jain, R. K.; Martin, J. D.; Stylianopoulos, T. The Role of Mechanical Forces in Tumor Growth and Therapy. Annual Review of Biomedical Engineering 2014, 16, 321-346, PMID: 25014786.

[27] Rauzi, M.; Lenne, P.-F. Cortical forces in cell shape changes and tissue morphogenesis. Curr. Top. Dev. Biol. 2011, 95, 93-144.

[28] Hutson, M. S.; Tokutake, Y.; Chang, M.-S.; Bloor, J. W.; Venakides, S.; Kiehart, D. P.; Edwards, G. S. Forces for morphogenesis investigated with laser microsurgery and quantitative modeling. Science 2003, 300, $145-149$.

[29] Boucher, Y.; Baxter, L. T.; Jain, R. K. Interstitial pressure gradients in tissue-isolated and subcutaneous tumors: implications for therapy. Cancer Res. 1990, 50, 4478-4484.

[30] Fadnes, H.; Reed, R.; Aukland, K. Interstitial fluid pressure in rats measured with a modified wick technique. Microvas. Res. 1977, 14, 27-36.

[31] Campàs, O.; Mammoto, T.; Hasso, S.; Sperling, R. A.; O'connell, D.; Bischof, A. G.; Maas, R.; Weitz, D. A.; Mahadevan, L.; Ingber, D. E. Quantifying cell-generated mechanical forces within living embryonic tissues. Nat. Methods 2014, 11, $183-189$.

[32] Dolega, M. E.; Delarue, M.; Ingremeau, F.; Prost, J.; Delon, A.; Cappello, G. Cell-like pressure sensors reveal increase of mechanical stress towards the core of multicellular spheroids under compression. Nat. Commun. 2017, 8, 14056-14064.

[33] Mohagheghian, E.; Luo, J.; Chen, J.; Chaudhary, G.; Chen, J.; Sun, J.; Ewoldt, R. H.; Wang, N. Quantifying compressive forces between living cell layers and within tissues using elastic round microgels. Nature Communications 2018, 9, 18781891.

[34] Gelimson, A.; Golestanian, R. Collective Dynamics of Dividing Chemotactic Cells. Phys. Rev. Lett. 2015, 114, 028101028105.

[35] Doering, C.; Mueller, C.; Smereka, P. Interacting particles, the stochastic Fisher-Kolmogorov-Petrovsky-Piscounov equation, and duality. Physica A: Statistical Mechanics and its Applications 2003, 325, 243-259.

[36] Dean, D. S. Langevin equation for the density of a system of interacting Langevin processes. J. Phys. A: Math. Gen. 1996, 29, L613-L617.

[37] Drasdo, D.; Höhme, S. A single-cell-based model of tumor growth in vitro: monolayers and spheroids. Phys. Biol. 2005, 2, 133-147.

[38] Sinha, S.; Malmi-Kakkada, A. N. Inter-particle adhesion regulates the surface roughness of growing dense three-dimensional active particle aggregates. Journal of Physical Chemistry B 2021, 125, 10445-10451.

[39] Sinha, S.; Li, X.; Das, R.; Thirumalai, D. Mechanical feedback controls the emergence of dynamical memory in growing tissue monolayers. arXiv:2202.04806 2021,

[40] Doostmohammadi, A.; Thampi, S. P.; Saw, T. B.; Lim, C. T.; Ladoux, B.; Yeomans, J. M. Celebrating Soft Matter's 10th Anniversary: Cell division: a source of active stress in cellular monolayers. Soft Matter 2015, 11, 7328-7336.

[41] Schaller, G.; Meyer-Hermann, M. Multicellular tumor spheroid in an off-lattice Voronoi-Delaunay cell model. Phys. Rev. E 2005, 71, 051910-051925.

[42] Pathmanathan, P.; Cooper, J.; Fletcher, A.; Mirams, G.; Murray, P.; Osborne, J.; Pitt-Francis, J.; Walter, A.; Chapman, S. A computational study of discrete mechanical tissue models. Phys. biol. 2009, 6, 036001-036014.

[43] Palsson, E.; Othmer, H. G. A model for individual and collective cell movement in Dictyostelium discoideum. Proc. Natl. Acad. Sci. 2000, 97, 10448-10453.

[44] Shraiman, B. I. Mechanical feedback as a possible regulator of tissue growth. Proc. Natl. Acad. Sci. 2005, 102, 3318-3323.

[45] Alessandri, K.; Sarangi, B. R.; Gurchenkov, V. V.; Sinha, B.; Kießling, T. R.; Fetler, L.; Rico, F.; Scheuring, S.; Lamaze, C.; Simon, A., et al. Cellular capsules as a tool for multicellular spheroid production and for investigating the mechanics of tumor progression in vitro. roc. Natl. Acad. Sci. 2013, 110, 14843-14848.

[46] Conger, A. D.; Ziskin, M. C. Growth of mammalian multicellular tumor spheroids. Cancer Res. 1983, 43, 556-560.

[47] Puliafito, A.; Hufnagel, L.; Neveu, P.; Streichan, S.; Sigal, A.; Fygenson, D. K.; Shraiman, B. I. Collective and single cell behavior in epithelial contact inhibition. Proc. Natl. Acad. Sci. 2012, 109, 739-744.

[48] Gniewek, P.; Schreck, C. F.; Hallatschek, O. Biomechanical Feedback Strengthens Jammed Cellular Packings. Phys. Rev. lett. 2019, 122, 208102-208107. 